Malignancy in patients with deep vein thrombosis-Of the 11 patients in the stocking group who developed deep vein thrombosis four had malignant disease (36\%), compared with six (27\%) of the 23 controls with deep vein thrombosis. Three patients who wore stockings $(27 \%)$ and nine controls $(39 \%)$ developed bilateral deep vein thrombosis (table II).

\section{Discussion}

Earlier trials of static compression for preventing deep vein thrombosis have been equivocal but the consensus of opinion has been that it is ineffective. In most trials, however, the compressive device has been badly designed. Usually elastic bandages or cylinders of elastic material-for example, Tubigrip -have been used. ${ }^{3}$ The compressive force applied to the leg by these methods varies greatly. In theory elastic bandages could be applied to take full account of the size and shape of the leg, but in practice their application cannot be standardised. The cylinders of elastic material are especially inappropriate as the lower limb is not cylindrical in shape but is more like an inverted cone. If cylindrical compression is applied to a cone-shaped structure the compressive force will be greatest at the cone's largest circumference-namely, the calf and thigh. The effect on the leg is much the same as that of applying a tourniquet at these sites.

Using graded compression, which takes account of the leg's conical shape and may be easily obtained by an appropriately designed elastic stocking, it is possible to reduce the incidence of deep vein thrombosis in patients undergoing major surgery from $49 \%$ to $23 \%$. The incidence in the control group was high, but this was accounted for by including only those patients undergoing major operations. There were too few patients to say whether static compression affected propagation of thrombus into the thigh or the incidence of pulmonary embolism. But
Wilkins ${ }^{8}$ reported in 1952 that static compression reduced the incidence of fatal pulmonary embolism fourfold. The higher incidence of malignant disease among the patients wearing stockings who developed deep vein thrombosis suggests that static compression may not be as effective in those with malignancy.

Despite possible defects in certain areas of deep vein thrombosis prevention with this method, a worthwhile reduction was observed. The stockings are easy to fit and need no maintenance after they are fitted. The stockings caused no complications and there were no complaints from patients or surgeons, largely because of the advance in design of compressive devices in recent years. Further investigation of the physiological and clinical effects of these devices is needed. Clinically this would best be met by a large scale trial reassessing their effect on pulmonary embolism.

I thank Professor A J Harding Rains, Mr B P Bliss, Mr A G Johnson, $\mathrm{Mr} \mathrm{J} \mathrm{E} \mathrm{H}$ Pendower, and $\mathrm{Mr} \mathrm{K} \mathrm{W}$ Reynolds for permission to study patients under their care; the nursing staff for their co-operation; Mrs S Jennings for technical help; and the Kendall Company, Barrington, Illinois, for providing the stockings.

Requests for reprints should be addressed to $\mathrm{Mr} \mathrm{C}$ P Holford, Department of Surgery, Charing Cross Hospital, London W6.

\section{References}

1 Tsapogas, M J, et al, Archives of Surgery, 1970, 101, 149.

2 Rosengarten, D S, et al, British fournal of Surgery, 1970, 57, 296.

${ }^{3}$ Browse, N L, et al, British fournal of Surgery, 1974, 61, 219.

4 Sigel, B, et al, Archives of Surgery, 1973, 106, 38.

5 Sigel, B, et al, Archives of Surgery, 1975, 110, 171.

${ }^{6}$ Lewis, F C, et al, Southwestern Surgical Congress, 3-6 May, 1976.

7 Kakkar, V V, et al, Lancet, 1970, 1, 540.

${ }^{8}$ Wilkins, R W, et al, New England fournal of Medicine, 1952, 246, 360.

\title{
Changes in nystagmus on raising body temperature in clinically suspected and proved multiple sclerosis
}

\author{
J V JESTICO, P D M ELLIS
}

British Medical fournal, 1976, 2, 970-972

\section{Summary}

Electronystagmography was used to record the appearance or alteration of nystagmus after raising the body temperature by a heat cradle. Nystagmus was increased or provoked in 13 out of 15 patients with clinically proved multiple sclerosis, in eight out of 12 suspected cases, but in no normal controls. No positive results were obtained in 12 patients with other neurological diseases, even when nystagmus was part of the clinical picture. The technique is simple to use and may have a role in the diagnosis of multiple sclerosis.

\footnotetext{
National Hospital for Nervous Diseases, Queen Square, London WC1N 3BG

J V JESTICO, BSC, MRCP, research registrar

Ferens Institute of Otolaryngology, Middlesex Hospital, London W1N 8AA

P D M ELLIS, FRCS, senior registrar
}

\section{Introduction}

An increase in body temperature in multiple sclerosis often causes a transient deterioration in the clinical state. ${ }^{1}$ This may be accompanied by an exacerbation of existing neurological signs and, in some cases, the appearance of new physical signs, which resolve as the temperature returns to normal. ${ }^{2-4}$ Up to now the somewhat inconvenient "hot-bath" test has been widely used in diagnosis in cases of suspected multiple sclerosis. We have developed a simple quantitative technique for studying the appearance or alteration of nystagmus induced by changes in body temperature and are at present assessing its value in the clinical diagnosis of multiple sclerosis. We report here our preliminary results in cases of clinically proved and suspected multiple sclerosis as well as in some other neurological disorders.

\section{Patients and method}

Thirty-nine patients and 10 normal controls were studied. Of the patients, 15 had clinically definite multiple sclerosis, 12 suspected multiple sclerosis, and 12 other neurological diseases. The controls comprised a man and a woman in each of the third, fourth, fifth, sixth, and seventh decades of life. 
Recordings were made at the National Hospital, Queen Square, and at the Ferens Institute. Two machines were used with AC amplification-a Devices single-channel pen recorder R2A with an S.8 coupling unit (calibrated to $1 \mathrm{~cm}$ deflection with the patient looking at an object at $1.8 \mathrm{~m}, 30$ degrees from the midline; time constant $7.5 \mathrm{~s}$; paper speed $1 \mathrm{~cm} / \mathrm{s}$ ); and an Elema-Schönander Junior ink-jet electroencephalograph (calibrated to $1 \mathrm{~cm}$ deflection/ $100 \mu \mathrm{V}$; time constant $0.3 \mathrm{~s}$; paper speed $15 \mathrm{~mm} / \mathrm{s}$ ). Silver silverchloride electrodes were placed at each outer canthus to record horizontal eye movements. It was arranged so that deviation of the eyes to the right produced an upward deflection on the paper. Before and after raising the body temperature 30-second recording samples were taken, both with the eyes open and with them closed, in the following positions of gaze: straight ahead, to the right, and to the left.

The electronystagmograms (ENGs) were reviewed by one of us (PDME) without access to the clinical data. The result was regarded as positive if after raising the body temperature nystagmus of the central type appeared or if there was an increase in frequency or amplitude of existing nystagmus. Central nystagmus occurs with disease affecting vestibular pathways and connections within the central nervous system. It is characteristically multidirectional and is not enhanced by loss of fixation.

Body temperature was raised by a standard heat cradle, in which electric lamps provided the heat source. The patient wearing underclothes or pyjamas lay on a couch, and trunk and limbs were covered with a thick blanket. This is important in patients with sensory loss as a precaution against burns from accidental contact with the lamps. The heat cradle was then placed over the patient's trunk and arms, and both patient and apparatus were covered with further layers of blankets so that only the patient's head was exposed. After the baseline oral temperature and an ENG had been recorded and the electric lamps turned on further temperature readings were taken at fiveminute intervals until a rise of $1^{\circ} \mathrm{C}$ had been achieved. The lamps were then switched off and the final ENGs recorded.

\section{Results}

Patients with clinically definite multiple sclerosis-In each case of clinically definite multiple sclerosis the diagnosis was based on the criteria of McAlpine et al. ${ }^{5}$ Seven of these patients had nystagmus at normal body temperature, and in all of them it was increased by heating (fig 1). Of the remaining eight patients who had no nystagmus initially, six developed it after heating (fig 2) and in two no change occurred.

Patients with suspected multiple sclerosis-These patients were classified as either probable or possible cases of multiple sclerosis according to the criteria of McAlpine et al. ${ }^{5}$ Five of the patients had nystagmus at normal body temperature, and in all of them it was increased by heating. Of the remaining seven patients with no nystagmus initially, three developed it after heating and in four no change occurred.

Patients with other neurological diseases-Of the 12 patients with other neurological diseases, six had nystagmus at normal body temperature. These were one with spinocerebellar degeneration, one with a posterior fossa meningioma, one with a pontine glioma, one with an acoustic neuroma, and two epileptics with drug-induced nystagmus.

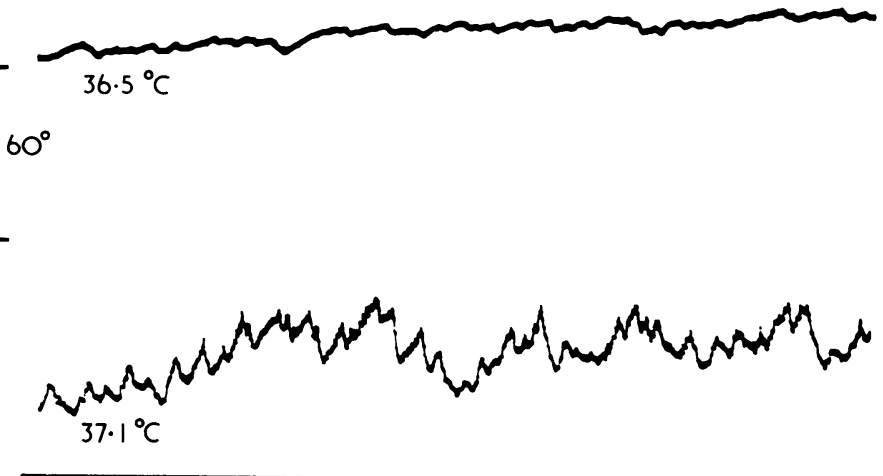

Time in seconds

FIG 1-ENG from patient with clinically definite multiple sclerosis showing increased nystagmus after raising body temperature.

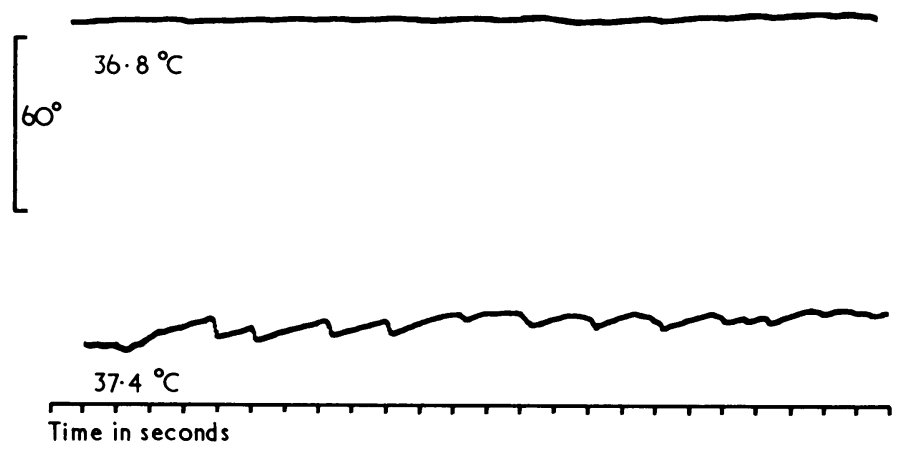

FIG 2-ENG from patient with clinically definite multiple sclerosis showing appearance of nystagmus after raising body temperature.

In addition, there were three patients who had nystagmus after stapedectomy. None of these patients had an increase in nystagmus on body heating. The remaining three patients-two with Parkinson's disease and one with torsion dystonia-had no nystagmus either before or after heating.

Controls-None of the controls had nystagmus either before or after heating.

\section{Discussion}

Multiple sclerosis is often diagnosed clinically from a history of relapsing and remitting neurological symptoms and physical signs indicating lesions disseminated in both space and time. It may also be suspected in some patients who present with a single neurological lesion-for example, a spastic paraparesis. The diagnosis can be made with confidence, however, only if additional discrete neurological lesions can be shown. Thus the study of visual evoked responses by the method of Halliday et $a l^{6}$ has proved to be a valuable adjunct in the clinical diagnosis. The finding of delayed responses, indicating a lesion within the visual pathways, often makes more-invasive procedures, including myelography, unnecessary. More recently, abnormalities of auditory evoked responses have been shown in patients with multiple sclerosis. ${ }^{7}$ There is clearly a need to develop other techniques aimed at demonstrating further otherwise silent lesions in areas of the nervous system known to be commonly affected by the disease.

Nystagmus is one of the most common physical signs in multiple sclerosis. Carter et al, ${ }^{8}$ reviewing a series of cases proved at necropsy, found it in up to $73 \%$ of patients at some time during the course of their disease. Our aim has been to investigate this sign further. Routine examination can give only a subjective impression of amplitude and speed and small changes may be missed. Our use of electronystagmography, on the other hand, has enabled us to obtain a permanent record of nystagmus and to assess quantitatively any changes that may occur with the eyes open or closed.

Rasminsky's ${ }^{9}$ elegant experiments in which diphtheria toxin was used to create demyelinating lesions in the ventral roots of rats showed that a conduction block can be produced across such lesions by raising the body temperature. Davis et al, ${ }^{10}$ studying experimental allergic neuronitis in pigs, recently confirmed that a similar reversible conduction block can be produced in demyelinating lesions of the peripheral nerves. Hence reversible impairment of nerve conduction with temperature appears to be a characteristic feature of demyelination. This phenomenon may partly explain the transient deterioration experienced by patients with multiple sclerosis when their temperature rises.

In our cases of clinically definite multiple sclerosis two positive phenomena occurred. Firstly, those patients found to have nystagmus on routine examination all showed an increase in nystagmus after a rise in body temperature. Secondly, many patients without nystagmus initially developed it on body heat- 
ing. Possibly more patients would have shown an abnormality had vertical nystagmus also been recorded.

Similar phenomena were seen in the patients with suspected multiple sclerosis. In these patients no definite diagnosis could be made as there was no evidence of more than one discrete neurological lesion. Some of these patients were found to have nystagmus on routine examination and so clearly had evidence of brain-stem dysfunction. These all showed an increase in nystagmus on body heating. Although this is not evidence of a further neurological lesion, we think that it is highly suggestive that the lesion is one of demyelination. In those in whom there was no initial nystagmus three out of seven developed it on heating. We believe that the appearance of central nystagmus produced by raising body temperature is evidence of previously unrecognised lesions affecting the vestibular and oculomotor connections within the brain stem.

Apart from multiple sclerosis many other diseases affecting the central nervous system also result in myelin breakdown. Not surprisingly, therefore, various physical signs, including weakness, reflex changes, and impairment of visual acuity, have been reported when patients with other neurological diseases are heated. Using the hot-bath test, Nelson et al ${ }^{11}$ described the appearance and increase of nystagmus in 33 out of 84 patients studied. Their positive results were obtained in nine patients with multiple sclerosis; 12 with epilepsy, most of whom were receiving anticonvulsants; 10 with "diffuse diseases of the central nervous system"; one with labyrinthitis; and, surprisingly, one with compression of the cauda equina. They also noted that in multiple sclerosis these changes occurred earlier and with smaller increases in body temperature. As yet we have had no positive results in patients with diseases other than multiple sclerosis, even though most of them had nystagmus.

From our encouraging initial results in patients with proved and suspected multiple sclerosis we think that recording nystagmus before and after raising the body temperature will prove to be a practicable diagnostic procedure. In cases of spastic paraparesis abnormal visual evoked responses are important as they indicate abnormalities outside the pyramidal pathways. The appearance of nystagmus on body heating in such cases, however, may not necessarily be due to a further discrete neurological lesion. This technique, therefore, may prove to be most valuable in such cases when a definite level below the brain stem can be identified. The investigation may also be important if the clinical history and physical examination do not suggest an abnormality within the brain stem-for example, acute retrobulbar neuritis.

The equipment needed to record visual evoked responses is not yet available in all neurological centres. On the other hand, the equipment necessary for recording nystagmus and that for heating the patient-namely, an electroencephalograph and a neurological heat cradle-are in current use. The technique is simple, causes little discomfort to the patient, and may be performed in the electroencephalography department, in the ward, or in the outpatient clinic. Our results suggest that it will provide useful additional information in patients suspected of having multiple sclerosis.

We thank Dr C J Earl and Dr M J G Harrison for allowing us to study their patients and for advice on preparing this paper; and Mr J Leech, of the MRC Hearing and Balance Unit, National Hospital, for technical advice.

Requests for reprints should be addressed to: Dr John Jestico, National Hospital for Nervous Diseases, Queen Square, London WC1N 3BG.

\section{References}

${ }^{1}$ Simons, D J, Bulletin of the Neurological Institute of New York, 1937, 6, 385. ${ }^{2}$ Guthrie, T C, Archives of Neurology and Psychiatry, 1951, 65, 437.

${ }^{3}$ Edmund, J, and Fog, T, Archives of Neurology and Psychiatry, 1955, 73, 316.

${ }^{4}$ Nelson, D A, and McDowell, F, fournal of Neurology, Neurosurgery, and Psychiatry, 1959, 22, 113.

${ }_{5}$ McAlpine, D, Lumsden, C E, and Acheson, E D, Multiple Sclerosis: a reappraisal, 2nd edn, p 202. Edinburgh, Churchill Livingstone, 1972.

${ }^{6}$ Halliday, A M, McDonald, W I, and Mushin, J, British Medical fournal, 1973, 4, 661 .

${ }^{7}$ Robinson, K, and Rudge, P, Lancet, 1975, 1, 1164.

${ }^{8}$ Carter, S, Sciarra, D, and Merritt, H H, Association for Research in Nervous and Mental Disease Proceedings, 1950, 28, 471.

9 Rasminsky, M, Archives of Neurology, 1973, 28, 287.

${ }^{10}$ Davis, F A, et al, fournal of Neurology, Neurosurgery, and Psychiatry, $1976,39,442$.

${ }_{11}$ Nelson, D A, Jeffreys, W H, and McDowell, F, Archives of Neurology and Psychiatry, 1958, 79, 31.

\title{
Can phenformin-induced lactic acidosis be prevented?
}

\author{
E A M GALE, R B TATTERSALL
}

and should not have been given phenformin. Four had hypertension and minimal evidence of renal disease, while in two no predisposing factor was identified. There are so many contraindications to the use of phenformin that it is doubtful whether patients on the drug can be monitored adequately. We suggest that phenformin should be withdrawn from general use.

Although patients taking phenformin are more likely to vascular, or hepatic disease, criteria for safe use of the drug are not well established. Eight diabetics died of lactic acidosis in Nottingham in 1972-5 and all were taking phenformin in therapeutic doses. Six had attended the diabetic clinic within a month of their terminal illness. Two patients had appreciable renal impairment

General Hospital, Nottingham NG1 6HA

E A M GALE, MRCP, registrar

R B TATTERSALL, MD, MRCP, consultant physician

\section{Introduction}

Soon after its introduction in 1957 phenformin was found to increase blood lactate concentrations. ${ }^{1}$ Nevertheless, the clinical syndrome of lactic acidosis seemed to be a rare complication, found only when other causes of lactate overproduction, such as shock, were present. In a comprehensive review in 1970 Oliva $^{2}$ could find only 18 published cases of phenforminassociated lactic acidosis. But the true incidence of this complication seems to have been seriously underestimated: in the last year over 50 cases have been reported from four centres. ${ }^{3-6}$ 